\title{
Smith-Lemli-Opitz syndrome and inborn errors of cholesterol synthesis: summary of the 2007 SLO/RSH Foundation scientific conference sponsored by the National Institutes of Health
}

\author{
Louise S. Merkens, PhD ${ }^{I}$, Christopher Wassif, $M S^{2}$, Kristy Healy, RN, CCRC $C^{l}$, \\ Anuradha S. Pappu, PhD ${ }^{3}$, Andrea E. DeBarber, PhD $D^{3}$, Jennifer A. Penfield, MS, PA-C ${ }^{1}$, \\ Rebecca A. Lindsay, BA $A^{4}$, Jean-Baptiste Roullet, $P h D^{1}$, Forbes D. Porter, MD, PhD', \\ and Robert D. Steiner, $M D^{1,5}$
}

\begin{abstract}
In June 2007, the Smith-Lemli-Opitz/RSH Foundation held a scientific conference hosted jointly by Dr. Robert Steiner from Oregon Health \& Science University and Dr. Forbes D. Porter from The Eunice Kennedy Shriver National Institute for Child Health and Human Development, National Institutes of Health. The main goal of this meeting was to promote interaction between scientists with expertise in cholesterol homeostasis, brain cholesterol metabolism, developmental biology, and oxysterol and neurosteroid biochemistry, clinicians researching and treating patients with Smith-Lemli-Opitz syndrome, the patient support organization and families. This report summarizes the presentations and discussions at the conference, represents the conference proceedings, and is intended to foster collaborative research and ultimately improve understanding and treatment of Smith-Lemli-Opitz syndrome and other inborn errors of cholesterol synthesis. Genet Med 2009: 11(5):359-364.
\end{abstract}

Key Words: Smith-Lemli-Opitz syndrome, cholesterol, hedgehog proteins, neurosteroids, cholesterol transport, mevalonate kinase deficiency, Niemann-Pick type C, oxysterols, CHILD syndrome

In June 2007, the Smith-Lemli-Opitz/RSH Foundation held a scientific conference hosted jointly by Dr. Robert Steiner from Oregon Health \& Science University (OHSU) and Dr. Forbes D. Porter from The Eunice Kennedy Shriver National Institute for Child Health and Human Development (NICHD), National Institutes of Health. The main goal of this meeting was to promote interaction between scientists with expertise in cholesterol homeostasis, brain cholesterol metabolism, developmental biology, and oxysterol and neurosteroid biochemistry, clinicians studying and treating patients with Smith-Lemli-Opitz syndrome (SLOS), patient support organization, and families. It was anticipated that these interactions could lead to collabora-

From the ${ }^{1}$ Department of Pediatrics, Oregon Health \& Science University, Portland, Oregon; ${ }^{2}$ Heritable Disorders Branch NICHD, NIH, DHHS, Bethesda, Maryland; Departments of ${ }^{3}$ Physiology and Pharmacology, ${ }^{4}$ Neurosurgery, and ${ }^{5}$ Molecular and Medical Genetics, Child Development and Rehabilitation Center, Oregon Clinical and Translational Research Center, and Doernbecher Children's Hospital, Oregon Health \& Science University, Portland, Oregon.

Louise S. Merkens, PhD, Department of Pediatrics, Oregon Health \& Science University (OHSU), Mail Code CDRC, 707 SW Gaines Road, Portland, OR 97239. E-mail: merkensl@ohsu.edu.

Disclosure: The authors declare no conflict of interest

Submitted for publication December 3, 2008.

Accepted for publication December 23, 2008.

DOI: $10.1097 /$ GIM.0b013e31819b246e tive research projects that would ultimately improve our understanding and treatment of SLOS and other inborn errors of cholesterol synthesis.

Several of the scientists also participated in the concurrent Smith-Lemli-Opitz/RSH Foundation family conference. These family sessions occur every 2 years as a forum for education of families with affected children about SLOS and dealing with children with a chronic disease. They are also an opportunity for SLOS families to meet and network. The families were invited to ask questions and express concerns to a panel of physicians, psychologists, dietitians, and scientists currently working in the diagnosis and management of patients with SLOS. This important event offered parents the opportunity to discuss current research with experts in the field.

The keynote speaker for the conference was Dr. G. Stephen Tint, who, in 1993, first identified the cholesterol biosynthetic defect causing SLOS. ${ }^{1}$ The keynote speaker award is presented as an honor to an individual with outstanding commitment to the field and is sponsored by the Smith-Lemli-Opitz/RSH Foundation Board. Dr. Tint's contributions and service to patients with SLOS and to their families are recognized internationally, and the hosts and sponsors of the conference were delighted that he was able to participate in this capacity despite recent retirement from his academic position.

SLOS is the most common among a group of disorders with multiple malformations and mental retardation due to defects in cholesterol synthesis. Mutations in the gene encoding the last enzyme, 7-dehydrocholesterol reductase, (DHCR7), result in low cholesterol and elevated concentrations of an abnormal sterol, 7-dehydrocholesterol (7DHC) in tissues and blood. SLOS has an observed incidence of at least 1 in 30,000 , but based on carrier rates it may be more common. The SLOS phenotype includes various congenital malformations, mental retardation, and distinctive autistic-like behavior. This group of human syndromes provides a unique opportunity to study the role of cholesterol synthesis and homeostasis in health, development, and behavior. SLOS may also turn out to be a treatable form of mental retardation if researchers are successful in their quest for effective treatments. Finally, learning more about cholesterol synthesis and metabolism by studying SLOS and related disorders will shed light on the more common disorders with hypercholesterolemia.

\section{CONFERENCE HIGHLIGHTS}

Sterols, development and the hedgehog pathway

Hedgehog $(\mathrm{Hh})$ proteins are signaling molecules that are secreted and function in diverse patterning of the development 
of body parts during embryogenesis. Hh proteins can promote cell proliferation, prevent apoptosis, and act as morphogens that specify cell responses depending on gradient tissue distribution.

As described by Dr. Beachy, newly synthesized Hh proteins undergo a series of posttranslational processing that involves covalent modification by cholesterol resulting in release of active signal with covalent cholesteryl adduct. ${ }^{2}$ Hh proteins are the only known proteins that are covalently modified by cholesterol. Even though 7DHC may be substituted for cholesterol in the activation of $\mathrm{Hh}$ proteins, reduction of total sterols, especially during in utero development, could interfere with $\mathrm{Hh}$ protein signaling and function. In addition to its role in the biogenesis of the Hh protein, cholesterol has an important role in response to the Hh protein signal. Cholesterol depletion limits the ability of cells to respond to the Hh protein.

In human CHILD syndrome and in bare patches ( $B p a)$ mice, there is a mutation in a sterol dehydrogenase (NSDHL) involved in cholesterol biosynthesis that results in defects in one or more developmental signaling pathways leading to male lethality, as described by Dr. Herman. In several Bpa alleles, the male lethality results from placental insufficiency. ${ }^{3,4}$ However, in vitro studies have shown that $7 \mathrm{DHC}$, desmosterol, and $7 \beta$ hydroxyl cholesterol can substitute for cholesterol in processing domain-mediated transfer reactions. Dr. Herman and coworkers have demonstrated expression of the ligand Indian hedgehog and the PTCH1 hedgehog receptor in mouse placenta. Affected placentas from the mutant mouse strain, $N s d h l^{B p a-8 a H}$, show decreased $\mathrm{Hh}$ signaling proteins in recipient placental cells of the allantoic mesoderm. ${ }^{4}$

\section{Effects of changes in cholesterol synthesis and absorption}

Alterations in the cholesterol pathway affect not only the synthesis of cholesterol but also the synthesis of other products that branch off from the pathway. Evaluation of the effects of agents or genetic disorders that block or up-regulate enzymes in the cholesterol pathway provides insights into this complex system.

Mevalonate kinase is an early enzyme in the pathway. Mutations in the gene encoding mevalonate kinase cause mevalonic aciduria in a severe form of the disease and hyperimmunoglobulinemia D with periodic fever syndrome (HIDS) in a milder form. The homozygous mevalonate kinase knock-out mice, developed in Dr. Gibson's lab, die as embryos implying a key role of this enzyme in mouse embryogensis. ${ }^{5}$ The heterozygotes, however, survive and show immune dysfunction. This model provides an opportunity to understand the mechanism(s) connecting alteration of the cholesterol pathway and immune function. Partial inactivation of mevalonate kinase may result in the depletion of the isoprene metabolites that are essential for prenylation of key proteins or the changes in lipid microdomains (rafts) of cell surface signaling proteins.

Lanosterol is a regulatory cornerstone in cholesterol biosynthesis. In the cholesterol pathway, it is the first sterol with the four-ring structure characteristic of all sterols, steroids, and bile acids. Itraconazole is an antifungal agent that inhibits CYP3A4 isoenzyme. As described by Dr. Lütjohann, clinical doses of this drug increase serum concentrations of lanosterol and dihydrolanosterol, lowering the LDL/HDL cholesterol ratio and potentially lowering liver degradation of 24S-hydroxycholesterol. ${ }^{6}$ Presenilins, endogenous proteins, also have effects on the lipid and protein composition of cellular membranes. Fibroblasts from presenilin double knock-out mice demonstrate lower lanosterol concentrations and up-regulated cholesterol synthetic enzymes. ${ }^{7}$
In SLOS, the last enzyme of the cholesterol pathway is inhibited resulting in low concentrations of cholesterol and high concentrations of 7DHC. To reduce synthesis of abnormal sterols, high-cholesterol diets and high-cholesterol diets with statin drugs (inhibitors of 3-hydroxy-3-methyl-glutaryl-CoA reductase) are being administered to determine their effectiveness as treatment. It is important to evaluate their effects on the cholesterol synthesis pathway as well as on the absorption of dietary cholesterol. Complex techniques using stable isotopes and gas chromatography-isotope ratio mass spectrometry ${ }^{8}$ are being used by Dr. Jones to study metabolism in children undergoing these treatments. Preliminary results show that highcholesterol diet with or without statins decreases the fractional cholesterol synthesis compared with very low cholesterol diet. In addition, there is a trend in the high-cholesterol diet with statins to decrease dietary cholesterol absorption compared with high-cholesterol diet without statins. ${ }^{9}$

Any therapies for SLOS involving dietary cholesterol need to take into account the extent of cholesterol absorption from the intestines. There are many factors that have the potential to affect cholesterol absorption; these include genetic factors, biliary cholesterol secretion, and conversion of cholesterol to bile acids. In addition, there are quantitative and qualitative differences in bile acids. Effects of bile acids were reviewed by Dr. Heubi. Some bile acids have no effect on cholesterol absorption, whereas others increase cholesterol absorption. It has also been shown that cholesterol absorption is dependent on luminal bile acids and micellar solubilization. ${ }^{10}$ In SLOS, urinary bile acids have been shown to be reduced compared with healthy children. ${ }^{11}$ Minimal information is available about luminal bile acid content in SLOS.

\section{Brain sterol metabolism}

As lipoproteins carrying cholesterol are not transported across the blood-brain barrier (BBB), cholesterol in the central nervous system (CNS) is almost entirely from de novo synthesis. Under steady-state conditions, an equivalent amount of cholesterol must be eliminated from the brain, and oxysterols effectively perform as transport forms of cholesterol.

A novel route for the elimination of brain oxysterols was presented by Dr. Meaney. In addition to the well-recognized passage of 24S-hydroxycholesterol across the BBB, there is also the passage of other oxysterols, most notably 27 -hydroxcholesterol, from the circulation into the brain. 27-Hydroxycholesterol seems to traverse the lipophillic BBB along a concentration gradient from the relatively high concentration in circulation to the brain. This is coupled with highly efficient metabolism to more polar metabolites within the brain; C27-cholestenoic acids, for example, are rapidly eliminated from the brain. Cholesterol 27-hydroxylase activity is deficient in the disorder cerebrotendinous xanthomatosis. Derangements in the pathway discussed by Meaney may affect brain cholesterol balance in cerebrotendinous xanthomatosis and contribute to the cerebral pathology. ${ }^{12}$

The role of 27-hydroxylation was described for SLOS, by Dr. Porter. ${ }^{13}$ In addition to the accumulation of $7 \mathrm{DHC}$ and its metabolites, levels of a novel oxysterol identified in vivo, 27hydroxy-7-dehydrocholesterol (27OH-7DHC), demonstrated significant negative correlation to cholesterol levels in plasma from SLOS patients. This suggests a role for this oxysterol in cholesterol homeostasis. As hedgehog signaling is impaired by low cholesterol, increased 27OH-7DHC may have detrimental effects during development by suppression of cholesterol levels. This hypothesis was tested by generating SLOS mice $\left(\right.$ Dhcr $\left.7^{-1-}\right)$ expressing a CYP27 transgene. These mice have 
significantly reduced tissue cholesterol/sterol levels and have a mutant phenotype that is more representative of severe human SLOS compared with littermate Dhcr $7^{-1-}$ embryos.

Cholesterol balance across the CNS and cholesterol turnover within the brain were reviewed by Dr. Dietschy. ${ }^{14}$ Cholesterol in the brain is present in two pools (1) cholesterol in the plasma membranes of glial cells and neurons and (2) cholesterol in the specialized membranes of myelin. Cholesterol turnover from these pools is normally low but may increase among glial cells and neurons during brain growth and neuron repair and remodeling. Internal recycling of cholesterol involves apolipoproteins such as apoE and apoA1, and one or more membrane transport proteins such as members of the low-density lipoprotein receptor family.

In the work presented by Dr. Vance, a population of lipoproteins secreted by glia, containing apoE and cholesterol, stimulated axon growth of CNS neurons. In addition, neurons were protected from apoptosis when apoE lipoproteins were bound to the low-density lipoprotein-receptor-related protein. Cholesterol turnover is known to be increased across the BBB for a number of neurodegenerative disorders, but alteration in sterol turnover within the brain that may in turn affect neuron and myelin integrity, has not been well studied. Niemann-Pick type $\mathrm{C}$ (NPC) disease is due to gene mutations in NPC1 or NPC2 that cause aberrant intracellular trafficking of cholesterol and result in accumulation of cholesterol in late endosomes/lysosomes. Other data showed that NPC1 deficiency in mouse neurons increased the cholesterol content of cell bodies but reduced cholesterol in distal axons. NPC1 protein is abundant in distal axons - in recycling endosomes in the presynaptic terminal. In addition to the role of NPC1 in cholesterol export from lysosomes, the findings suggest a neuron-specific role for NPC1 in the synaptic vesicle recycling pathway that may contribute to the severe neurological phenotype characteristic of NPC disease. ${ }^{15}$

\section{Prenatal and postnatal diagnosis of SLOS}

Accurate measures of incidence and early diagnosis of SLOS are important to establish the scope of this disorder. Diagnosis in utero is a likely prerequisite toward optimizing interventions to increase cholesterol transport to an affected fetus.

Methods of prenatal diagnosis of SLOS were discussed by Dr. Roberson. An SLOS risk screening algorithm based on the common maternal serum triple screen for congenital defects (alpha-fetoprotein, human chorionic gonadotropin, and unconjugated estriol) was implemented at 15 prenatal screening programs. ${ }^{16}$ Diagnostic testing of amniotic fluid was offered to those with positive screens (risk $\geq 1: 50$ ). Of 1,079,301 pregnancies evaluated for risk, 3,083 were screen positive, and five pregnancies were ultimately diagnosed with SLOS. The prevalence of SLOS in second trimester pregnancies was 1:101,000. The pregnancies identified as high risk by screening that were not affected by SLOS were associated with high risk for other major fetal abnormalities.

A noninvasive method using maternal serum and urine to identify only those steroids produced in pregnancies with SLOS affected fetuses was also developed. ${ }^{17}$ The steroid ratios determined to be diagnostic were 7-dehydropregnanetriol/pregnantriol (7-PT/PT), 8-dehydropregnanetriol/PT (8-PT/PT), the sum of those two $((7+8)-\mathrm{PT} / \mathrm{PT})$, and dehydroestriol/estriol. Dr. Shackleton evaluated those methods that he developed in a multicenter trial. Initial screening used the algorithm described above. This identified 737 pregnancies at risk for SLOS, of which five were positive for SLOS by amniotic fluid measurement and retroactively by urine and serum steroid ratio quanti- tation. It was determined that after the 15 th week of gestation, either urine or serum steroid ratios are diagnostic, with urine a more definitive test.

Dr. Krajewska-Walasek's laboratory at the only health center in Poland where diagnosis of SLOS has been performed for the past 15 years has also implemented a new diagnostic procedure using urine. ${ }^{18}$ Maternal urinary steroids 7DHPT and 8DHE3 are being used for prenatal diagnosis in pregnancies suspected of SLOS. Using all methods of diagnosis, SLOS has been identified by this institute in 71 patients and 20 prenatal cases during the past 15 years. The laboratory has identified 18 different DHCR7 mutations, with two (c.452G $>$ A and c.976C $>$ T) accounting for $65.2 \%$ of mutations. Population screening for these two mutations among 4256 neonates indicated a carrier frequency of $2.4 \%$, which would predict an incidence of SLOS between 1:2300 and 1:3937, making it among the most common recessive genetic disorders in Poland. The actual incidence in Poland is currently under investigation using a population-based registry, monitoring 300,000 births/year in all Polish provinces.

The predicted incidence of SLOS in the North American white population has been calculated from carrier frequency of the common mutation, IVS $8-1 \mathrm{G}>\mathrm{C}$. Allele frequencies were found to be between $1: 1,590$ and 1:17,000. ${ }^{19,20}$ The observed prevalence and incidence are lower. ${ }^{21}$ The discrepancy between expected and observed incidence can be explained in part by prenatal, neonatal, and infant deaths of the most severely affected children, and under ascertainment of mild and atypical cases. According to Dr. Nowaczyk, recent observations put the estimate of SLOS prevalence at 16 weeks of gestation similar to that observed at birth $(\sim 1: 60,000)$, suggesting that reduced fertility of carrier couples or fetal loss in the first trimester play a significant role in explaining the discrepancy. It is also possible that population screening for the most common mutation may not reflect true carrier rates.

\section{Effect of SLOS on embryonic development and placental transport of cholesterol}

Maternal cholesterol, synthesis of cholesterol in the fetus, and the transport of cholesterol during fetal development were discussed. These areas of research may lead to improved placental transport of cholesterol as a novel therapeutic intervention.

Dr. Tint has done extensive research to describe the differences in cholesterol synthesis and sterol accumulation in different organs of the developing mouse fetus. ${ }^{22}$ The SLOS knockout mouse, however, shows a phenotype different from humans with SLOS, and the placental structures responsible for transporting cholesterol from mother to fetus are also different in the two species. Thus, a better animal model is needed to learn more about the role of cholesterol transport and cholesterol synthesis in the fetus. A model of the pregnant rat that is treated with an inhibitor of DHCR7 may be more useful.

In addition to evaluating cholesterol synthesis in the brain, Dr. Tint has identified the likely mechanism causing the relatively high concentrations of desmosterol in fetal brain. There is a short sequence identified as a neuron-restrictive silencer element in the promoter region of the gene for 24-dehydrocholesterol reductase (the enzyme that converts desmosterol to cholesterol) that binds to the regulatory protein NRSF/REST (neuron-restrictive silencing factor/repressor element1 silencing transcription factor) as a transcriptional enhancer. Because the expression of REST is naturally reduced in brain compared with most other tissues, this results in a down-regulated state with reduced enzyme activity and accumulation of desmosterol. 
Using the hamster, Dr. Woollett has demonstrated an increase in fetal cholesterol when maternal cholesterol concentrations are elevated with diet. ${ }^{23}$ In vitro studies using rodent yolk sac and human-derived choriocarcinoma BeWo cells also demonstrated a relationship between "maternal" cholesterol concentrations and output of cholesterol to the "fetus."24,25 Data have shown that the HDL isolated from fetal cord blood from an SLOS fetus effluxed about 50\% more cholesterol from BeWo cells ${ }^{26}$; this suggests that the amount of cholesterol transported can change depending on the acceptor protein. The ability to manipulate the mass of maternal cholesterol that crosses to the fetus could improve fetal development and improve outcome in those with SLOS. 27

Maternal cholesterol is essential for hormonal and physical changes of pregnancy. Dr. Muenke's laboratory studied motherinfant pairs (9938) to assess whether low maternal serum cholesterol during pregnancy is associated with adverse birth outcomes. $^{28}$ They found an increased prevalence of preterm delivery and lower birth weights of term infants from mothers with low total cholesterol ( $<10$ th percentile). There was a trend toward microcephaly, but no increase in congenital anomalies. These results may be mediated by the decrease in substrate for hormonal and nutritional support of early pregnancy. Or perhaps the results are due to altered lipoproteins that have been shown to change pregnancy outcome in other studies. Whatever the reasons, the associations emphasize the importance of cholesterol for optimal fetal development.

\section{Novel treatment of SLOS and related disorders}

The behavioral and learning problems in SLOS are likely due to a combination of neurodevelopmental deficits, and functional deficits as a result of abnormal sterol composition in the CNS. Although neurodevelopmental deficits may be permanent, functional deficits can potentially be treated. Since dietary cholesterol does not cross the $\mathrm{BBB}$, various approaches are being considered to increase CNS cholesterol or to correct the biochemical disturbances that affect neurological function.

One approach to improve the biochemical abnormality in SLOS is gene therapy. Dr. Watson and coworkers are using the mouse models developed by Dr. Porter's group to evaluate the efficacy of adeno-associated viral (AAV) therapy in treating both peripheral organs and the CNS. Since cholesterol can be transferred between cells, a major question in these studies is whether product (i.e., cholesterol) cross-correction will occur between treated cells and untreated cells. Dr. Watson presented preliminary data showing that DHCR7-AAV vectors can be used to express $D H C R 7$ in mouse liver tissue, and that this therapy resulted in an improved serum dehydrocholesterol/cholesterol ratio. Dr. Watson's group has developed techniques for treatment of the CNS with AAV vectors in newborn mice. They will now apply these techniques to investigate the efficacy of gene therapy in SLOS mice. Since neurodevelopmental defects occur before birth, future studies will also investigate prenatal therapy.

The intracellular accumulation of 7DHC in SLOS can have detrimental effects. Drs. Lloyd-Evans and Platt have now clearly confirmed that a secondary defect in intracellular cholesterol transport occurs in SLOS cells. There is a similar defect in intracellular transport in NPC1-mutant cells. ${ }^{29}$ Multiple experiments demonstrated other similarities between SLOS and NPC. These include defects in endosomal transport, deficient lysosomal calcium pools, and glycosphingolipid (GSL) accumulation. In SLOS the accumulation of GSLs increases with increased disease severity. Treatment of SLOS cells, in vitro, with an inhibitor of GSL biosynthesis, miglustat, decreased the abnormal accumulation of GSLs and reversed the defect in the intracellular transport of cholesterol. It is possible that this defect in intracellular cholesterol transport in SLOS exacerbates the cholesterol synthetic defect by decreasing the bioavailability of cholesterol. Thus, it is plausible that inhibition of GSL synthesis could increase cholesterol bioavailability, and thus be a novel therapeutic intervention for SLOS. Future studies in mouse models of SLOS are planned.

Although dietary cholesterol does not cross the BBB, anecdotal evidence suggests that behavior improves in SLOS patients on dietary cholesterol supplementation. In contrast to cholesterol, neuroactive steroids do cross the BBB and could potentially influence the SLOS behavioral phenotype. In NPC, it has been proposed that a deficiency of allopregnanolone (a neurosteroid) may be a contributing factor in the pathogenesis of this neurodegenerative disorder. Previous studies demonstrated that allopregnanolone therapy significantly increased the lifespan of NPC mice. Dr. Ory's work supports the idea that allopregnanolone acts through a non-GABAa receptor-dependent mechanism, possibly through activation of pregnane $\mathrm{X}$ receptor-dependent pathways. Furthermore, therapy combining allopregnanolone with a liver $\mathrm{X}$ receptor-agonist may provide additional benefit. Although these studies were focused on understanding NPC, they may provide some insight as to how disturbed neurosteroid synthesis may contribute to the pathology of SLOS, and how correction of a defect in neurosteroid synthesis could be of potential therapeutic benefit in SLOS.

\section{Intracellular and membrane effects in SLOS: fibroblasts and retina}

The genetic and phenotypic profiles of SLOS have been well described. New advances, however, are being made in characterizing the biochemical and cellular defects in this disorder using in vitro and in vivo models and human patient studies.

Dr. Tulenko, for example, compared human fibroblasts obtained from both patients and healthy controls. It has been shown that not only is the cellular membrane composition significantly altered in SLOS fibroblasts, but also that these alterations have profound effects on the membrane electrophysiology, protein expression in the membrane, and downstream cellular signaling. ${ }^{30}$ In particular, the marked deficit of cholesterol and the increased accumulation of 7DHC in the biological membrane that alter the membrane function seem to suggest abnormal caveolar function. SLOS cells are transcriptionally deficient in the cholesterol binding, scaffolding, and cellular signaling caveolar protein caveolin-1, which may be a direct result of the altered bilayer organization. One consequence of the caveolin-1 deficiency seems to be a reduction in the $\mathrm{BKCa}$ protein, a protein that normally colocalizes with caveolin-1, resulting in suppression of BKCa-mediated outward $\mathrm{K}^{+}$currents and globally impaired membrane electrophysiology. Another consequence is structural defects due to disrupted actin distribution in response to decreased caveolin-1 levels.

In addition to this in vitro work, studies done with isolated whole retinas from rats treated with AY9944 (a selective inhibitor of DHCR7, which produces an animal model that mimics key features of the human disease) have shown that hundreds of genes involved in multiple biochemical pathways are significantly affected. In addition, the levels of glycerophospholipid molecular species containing docosahexaenoic acid in retinas from treated animals are markedly lower than in age-matched controls, ${ }^{31}$ and the treated retinas also contain oxidized lipids and oxidized proteins. These studies, carried out by Dr. Fliesler, suggest that beyond the initial defect in cholesterol biosynthesis, 
global metabolic alteration involving cross-talk between the cholesterol pathway and other metabolic pathways as well as lipid and protein oxidation likely contribute to the retinal dysfunction and pathogenesis observed in the AY9944 rat model of SLOS $^{32}$ and may have direct relevance to underlying pathogenic mechanisms in SLOS.

Complementing this work, human electroretinogram studies were performed in SLOS patients by Dr. Elias. Most patients exhibited abnormal electroretinograms showing scotopic and photopic (rod and cone) aberrancy. ${ }^{33,34}$ Several of these patients also had abnormally formed optic nerves, retinal pigmentary deposits, early nuclear cataracts, and/or a reduction in visual acuity, supporting in vivo descriptions of retinal dysfunction. Although many of the neurological symptoms of SLOS are still waiting for a treatment to be developed, there is some evidence that high cholesterol diets supplemented with antioxidants may improve some of the retinal abnormalities found in SLOS.

\section{Family program}

The family program ran concurrently with the research presentations. For 2 days, the families were treated to sessions on stress management, behavior problems, sleep issues, development of the Individualized Education Program in school, reflux, nutrition, oral dysphasia and feeding problems, an overview of research studies, and puberty in girls, most of which were led by professionals who work with SLOS families on a regular basis. There was also socializing over meals and activities, roundtable discussions, and a workshop for siblings. On the third day, the families and the researchers came together for more presentations centering on clinical research studies, socialization over lunch and a lengthy question and answer session with the clinical researchers. The day ended with a large round table discussion between the SLO/RSH Foundation and the members. It was genuinely clear that the personal contact between families is critical for support. There is great comfort in knowing no explanations are needed while in the company of other SLOS families and the wealth of knowledge shared is immeasurable. It is also clear how important contact is between the families and researchers to validate why the research is needed and to brainstorm new theories and direction.

\section{AREAS OF FUTURE RESEARCH}

Although progress has been made to understand the pathology of SLOS and insights into potential treatments, there are many unanswered questions. Below is partial list of future areas of research.

- Develop better animal models to understand the pathology as well as to test treatments.

- Identify factors that enhance cholesterol absorption and retention.

- Understand the effect of deficient cholesterol synthesis and excess 7DHC on brain metabolism and function.

- Determine if maternal treatment with high cholesterol will prevent some of the anatomic alterations that often occur during development. Develop methods to enhance cholesterol transport across the placenta.

- Research areas that affect daily lives of children living with SLOS and families such as behavior, nutrition and feeding, sleep, gastrointestinal issues, communication, and autistic tendencies.

\section{ACKNOWLEDGMENTS}

This work was supported by the Grant awards: R01 HL073980 03S1 (R01 supplement) (to R.S.); from the intramural research programs of the Eunice Kennedy Shriver National Institute of Child Health and Human Development and the National Institutes of Health Office of Rare Diseases (to R.S. F.P.).

The authors thank Cindy Early, Cylia Amendolara, and Sylvia Hathaway in the OHSU Department of Pediatrics Business Office for their help with administrative details. Cindy Gold of the SLO/RSH Foundation was instrumental in the planning and implementation of the conference. They appreciate the assistance of Abby Ershow, program officer at NHLBI, and Lisa Deveaux of the NICHD administrative office.

\section{REFERENCES}

1. Tint GS, Irons M, Elias ER, et al. Defective cholesterol biosynthesis associated with the Smith-Lemli-Opitz syndrome. N Engl J Med 1994;330:107-113.

2. Cooper MK, Wassif CA, Krakowiak PA, et al. A defective response to Hedgehog signaling in disorders of cholesterol biosynthesis. Nat Genet 2003;33:508-513.

3. Caldas H, Cunningham D, Wang X, et al. Placental defects are associated with male lethality in bare patches and striated embryos deficient in the NAD(P)H Steroid Dehydrogenase-like (NSDHL) Enzyme. Mol Genet Metab 2005;84:48-60.

4. Jiang F, Herman GE. Analysis of Nsdhl-deficient embryos reveals a role for Hedgehog signaling in early placental development. Hum Mol Genet 2006; 15:3293-3305

5. Hager EJ, Tse HM, Piganelli JD, et al. Deletion of a single mevalonate kinase (Mvk) allele yields a murine model of hyper-IgD syndrome. $J$ Inherit Metab Dis 2007;30:888-895

6. Schneider B, Gerdsen R, Plat J, et al. Effects of high-dose itraconazole treatment on lipoproteins in men. Int J Clin Pharmacol Ther 2007;45:377-384.

7. Tamboli IY, Prager K, Thal DR, et al. Loss of gamma-secretase function impairs endocytosis of lipoprotein particles and membrane cholesterol homeostasis. J Neurosci 2008;28:12097-12106.

8. Vanstone CA, Raeini-Sarjaz M, Parsons WE, Jones PJ. Unesterified plant sterols and stanols lower LDL-cholesterol concentrations equivalently in hypercholesterolemic persons. Am J Clin Nutr 2002;76:1272-1278.

9. Chan YM, Merkens LS, Roullet JB, Connor WE, Steiner RD, Jones PJH. Effects of dietar cholesterol and simvastatin on cholesterol absorption and synthesis (CAS) in Smith-Lemli-Opitz syndrome (SLOS) [Abstract]. Washington, DC: FASEB Annual Meeting; 2007.

10. Woollett LA, Wang Y, Buckley DD, et al. Micellar solubilisation of cholesterol is essential for absorption in humans. Gut 2006;55:197-204.

11. Natowicz MR, Evans JE. Abnormal bile acids in the Smith-Lemli-Opitz syndrome. Am J Med Genet 1994;50:364-367.

12. Panzenboeck U, Andersson U, Hansson M, Sattler W, Meaney S, Bjorkhem I. On the mechanism of cerebral accumulation of cholestanol in patients with cerebrotendinous xanthomatosis. J Lipid Res May 2007; 48:1167-1174.

13. Wassif CA, Yu J, Cui J, Porter FD, Javitt NB. 27-Hydroxylation of 7- and 8-dehydrocholesterol in Smith-Lemli-Opitz syndrome: a novel metabolic pathway. Steroids 2003;68:497-502.

14. Dietschy JM, Turley SD. Thematic review series: brain Lipids. Cholesterol metabolism in the central nervous system during early development and in the mature animal. $J$ Lipid Res 2004;45:1375-1397.

15. Vance JE. Lipid imbalance in the neurological disorder, Niemann-Pick C disease. FEBS Lett 2006;580:5518-5524.

16. Craig WY, Haddow JE, Palomaki GE, et al. Identifying Smith-Lemli-Opitz syndrome in conjunction with prenatal screening for Down syndrome. Prenat Diagn 2006;26:842-849.

17. Shackleton CH, Roitman E, Kratz L, Kelley R. Dehydro-oestriol and dehydropregnanetriol are candidate analytes for prenatal diagnosis of SmithLemli-Opitz syndrome. Prenat Diagn 2001;21:207-212.

18. Jezela-Stanek A, Malunowicz EM, Ciara E, et al. Maternal urinary steroid profiles in prenatal diagnosis of Smith-Lemli-Opitz syndrome: first patient series comparing biochemical and molecular studies. Clin Genet 2006;69: 77-85.

19. Battaile KP, Battaile BC, Merkens LS, Maslen CL, Steiner RD. Carrier frequency of the common mutation IVS $8-1 \mathrm{G}>\mathrm{C}$ in DHCR7 and estimate of the expected incidence of Smith-Lemli-Opitz syndrome. Mol Genet Metab 2001;72:67-71.

20. Waye JS, Nakamura LM, Eng B, et al. Smith-Lemli-Opitz syndrome: carrier frequency and spectrum of DHCR7 mutations in Canada. J Med Genet 2002;39:E31. 
21. Nowaczyk MJ, Zeesman S, Waye JS, Douketis JD. Incidence of SmithLemli-Opitz syndrome in Canada: results of three-year population surveillance. J Pediatr. 2004;145:530-535.

22. Tint GS, Yu H, Shang Q, Xu G, Patel SB. The use of the Dhcr7 knockout mouse to accurately determine the origin of fetal sterols. J Lipid Res 2006; $47: 1535-1541$

23. McConihay JA, Horn PS, Woollett LA. Effect of maternal hypercholesterolemia on fetal sterol metabolism in the Golden Syrian hamster. J Lipid Res 2001;42:1111-1119.

24. Schmid KE, Davidson WS, Myatt L, Woollett LA. Transport of cholesterol across a BeWo cell monolayer: implications for net transport of sterol from maternal to fetal circulation. J Lipid Res 2003;44:1909-1918.

25. Hayden Lichtenberg M, Wilke CS, McConihay JA, Granholm NA, Woollett LA. Yolk sac cholesteryl ester secretion rates can be manipulated in the Golden Syrian hamster: effect of yolk sac cholesterol concentrations. Bioch Biophys Acta 2005;1735:214-221.

26. Jenkins KT, Merkens LS, Tubb MR, et al. Enhanced placental cholesterol efflux by fetal HDL in Smith-Lemli-Opitz syndrome. Mol Genet Metab 2008; $94: 240-247$

27. Woollett LA. Maternal cholesterol in fetal development: transport of cholesterol from the maternal to the fetal circulation. Am J Clin Nutr 2005;82: $1155-1161$.

28. Edison RJ, Berg K, Remaley A, et al. Adverse birth outcome among mothers with low serum cholesterol. Pediatrics. 2007;120:723-733.

29. Lloyd-Evans E, Morgan AJ, He X, et al. Niemann-Pick disease type C1 is a sphingosine storage disease that causes deregulation of lysosomal calcium. Nat Med 2008;14:1247.

30. Tulenko TN, Boeze-Battaglia K, Mason RP, et al. A membrane defect in the pathogenesis of the Smith-Lemli-Opitz syndrome. J Lipid Res 2006;47:134143.

31. Ford DA, Monda JK, Brush RS, Anderson RE, Richards MJ, Fliesler SJ. Lipidomic analysis of the retina in a rat model of Smith-Lemli-Opitz syndrome: alterations in docosahexaenoic acid content of phospholipid molecular species. J Neurochem 2008;105:1032-1047.

32. Fliesler SJ, Peachey NS, Richards MJ, Nagel BA, Vaughan DK. Retinal degeneration in a rodent model of Smith-Lemli-Opitz syndrome: electrophysiologic, biochemical, and morphologic features. Arch Ophthalmol 2004; 122:1190-1200.

33. Elias ER, Fulton A, Mayer DL, Hansen RM. Retinal dysfunction in patients with the Smith-Lemli-Opitz syndrome. Am J Hum Gen 2000;67 (4 suppl 2)(36 A132).

34. Elias ER, Hansen RM, Irons M, Quinn NB, Fulton AB. Rod photoreceptor responses in children with Smith-Lemli-Opitz syndrome. Arch Ophthalmol 2003; $121: 1738-1743$.

\section{APPENDIX}

\section{Conference participants}

Alka Aneja, Kennedy Krieger Institute, Johns Hopkins University School of Medicine, Baltimore, MD; Philip A. Beachy, Howard Hughes Medical Institute, Stanford CA; William E. Connor, Oregon Health \& Science University, Portland OR; John M. Dietschy, UT Southwestern Medical Center, Dallas, TX; Ellen R. Elias, University of Colorado Health Center, Denver, CO; Steven J. Fliesler, University at Buffalo/SUNY and VAWNY-Research Service, Buffalo, NY; K. Michael Gibson, Children's Hospital, University of Pittsburgh School of Medicine, Pittsburgh, PA; Gail E. Herman, Columbus Children's Research Institute \& Ohio State University, Columbus, OH; James E. Heubi, Cincinnati Children's Hospital Medical Center, Cincinnati, OH; Peter Jones, The Richardson Centre for Functional Foods and Nutraceuticals, University of Manitoba, Winnipeg, Manitoba, Canada; Malgorzata KrajewskaWalasek, University of Medical Sciences, Poznan Poland; Emyr Lloyd-Evans, University of Oxford, Oxford, United Kingdom; Dieter Lütjohann, University of Bonn, Bonn, Germany; Steven Meaney, Dublin Institute of Technology, Dublin, Ireland; Maximilian Muenke, National Human Genome Research Institute, Bethesda, MD; Malgorzata J. M. Nowaczyk, McMaster University, Hamilton, Ontario, Canada; Edward A. Neuwelt, Oregon Health \& Science University, Portland OR; Daniel S. Ory, Washington University School of Medicine, St. Louis, MO; Fran Platt, University of Oxford, Oxford, United Kingdom; Marie Roberson, State of California, Department of Health Service, Genetics Disease Branch CA; Cedric Shackleton, Children's Hospital, Oakland Research Institute, Oakland, CA; Elaine Tierney, Kennedy Krieger Institute, Johns Hopkins University School of Medicine, Baltimore, MD; G. Stephen Tint, VA Medical Center, East Orange NJ \& UMDNJ-New Jersey Medical School, Newark, NJ; Thomas N. Tulenko, Thomas Jefferson University, Philadelphia, PA; Jean E. Vance, University of Alberta, Edmonton, Alberta, Canada; Gordon Watson, Children's Hospital Oakland Research Institute, Oakland CA; Laura A. Woollett, University of Cincinnati Medical School, Cincinnati, OH. 